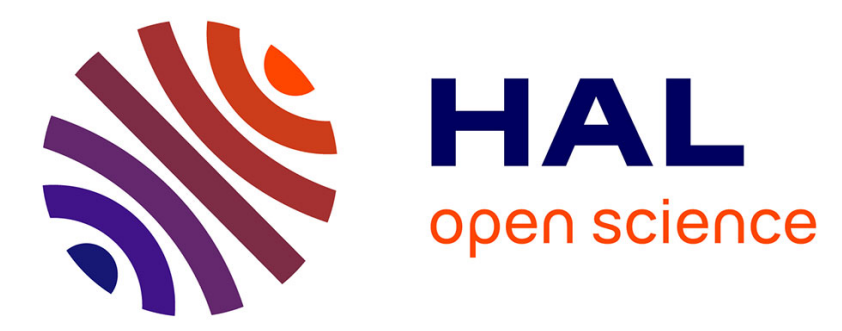

\title{
L'IPA dans le suivi respiratoire post-Covid-19
}

Pierre-Yves Blanchard, Anne-Marie Ruppert, Morgane Faure, Nathalie Nion

\section{To cite this version:}

Pierre-Yves Blanchard, Anne-Marie Ruppert, Morgane Faure, Nathalie Nion. L'IPA dans le suivi respiratoire post-Covid-19. Soins, 2021, 66 (853), pp.32-34. 10.1016/S0038-0814(21)00056-6 . hal03264043

\section{HAL Id: hal-03264043 \\ https: / hal.sorbonne-universite.fr/hal-03264043}

Submitted on 17 Jun 2021

HAL is a multi-disciplinary open access archive for the deposit and dissemination of scientific research documents, whether they are published or not. The documents may come from teaching and research institutions in France or abroad, or from public or private research centers.
L'archive ouverte pluridisciplinaire $\mathbf{H A L}$, est destinée au dépôt et à la diffusion de documents scientifiques de niveau recherche, publiés ou non, émanant des établissements d'enseignement et de recherche français ou étrangers, des laboratoires publics ou privés. 
$\underline{\text { Titre : }}$

L'IPA dans le suivi respiratoire post-Covid-19

\section{Résumé :}

La mise en place d'une activité de pratique avancée infirmière pour le suivi des patients atteints de formes sévères ou très sévères de pneumonie à severe acute respiratory syndrome coronavirus 2 permet de répondre aux besoins de santé nouveaux d'une population et à l'afflux massif de malades. Les compétences de l'infirmier en pratique avancée sont mobilisées afin d'assurer les missions de prévention et de dépistage de potentielles séquelles pouvant conduire à une insuffisance respiratoire chronique.

\section{$\underline{\text { Points à retenir : }}$}

- Le suivi respiratoire des patients atteints de formes sévères de Covid-19 répond à un besoin de santé nouveau et à l'afflux massif des patients

- En vue de dépister des séquelles respiratoires, l'infirmier en pratique avancée (IPA) a pour missions : le recueil de l'histoire de la maladie, l'interprétation des résultats des examens, la réalisation d'un entretien et de l'examen clinique, la pose de conclusions cliniques

- L'IPA répond aux interrogations des patients, donne des conseils éducatifs adaptés et explique les résultats de leurs examens

- Les données de suivi sont compilées par l'IPA dans un compte-rendu qui est adressé au patient et aux médecins impliqués dans le parcours de soins du patient

Mots-clés :

Covid-19 ; infirmière ; pneumologie ; pneumopathie virale ; pratique avancée

\section{Auteurs :}

Blanchard Pierre-Yves, infirmier en pratique avancée, groupe hospitalier AP-HP. Sorbonne Université, département médico-universitaire APPROCHES

Hôpital Tenon, 4 rue de la Chine, 75970 Paris cedex 20

Hôpital de la Pitié-Salpêtrière, 47-83 boulevard de 1'Hôpital, 75013 Paris

pierre-yves.blanchard@aphp.fr 
Ruppert Anne-Marie, maître de conférence des universités, praticien hospitalier, groupe hospitalier AP-HP. Sorbonne Université, département médico-universitaire APPROCHES

Hôpital Tenon, 4 rue de la Chine, 75970 Paris cedex 20

anne-marie.ruppert@aphp.fr

Faure Morgane, praticien hospitalier, groupe hospitalier AP-HP. Sorbonne Université, département médico-universitaire APPROCHES

Hôpital de la Pitié-Salpêtrière, 47-83 boulevard de l'Hôpital, 75013 Paris

morgane.faure@aphp.fr

Nion Nathalie, cadre paramédicale du Département Médico-Universitaire APPROCHES, groupe hospitalier AP-HP. Sorbonne Université, département médico-universitaire APPROCHES

Hôpital de la Pitié-Salpêtrière, 47-83 boulevard de l’Hôpital, 75013 Paris

nathalie.nion@aphp.fr

Les auteurs ne déclarent aucun lien d'intérêt en lien avec cet article.

Auteur correspondant : PY Blanchard pierre-yves.blanchard@aphp.fr 


\section{$\underline{\text { Article : }}$}

Le nouveau coronavirus severe acute respiratory syndrome coronavirus 2 (Sars-CoV-2) entraine chez certains patients une atteinte potentiellement grave nommée Covid-19. Cette maladie peut prendre différentes formes et affecter le système respiratoire, digestif, neurocognitif, hépatorénal et le derme. Des complications thromboemboliques ont également été décrites. Dans les formes sévères, la Covid-19 est à l'origine d'une pneumonie nécessitant une oxygénothérapie et une hospitalisation. Dans les formes les plus sévères, elle est responsable d'un syndrome de détresse respiratoire aiguë qui justifie une hospitalisation en soins intermédiaires (le plus souvent en soins intensifs) ou en réanimation pour un traitement par oxygénothérapie à haut débit ou une ventilation mécanique invasive.

Du fait de la nouveauté de cette maladie, l'état des connaissances actuelles ne permet pas d'établir si des séquelles peuvent persister à long terme. Néanmoins, la gravité des atteintes pulmonaires, tant sur le plan clinique, scannographique que fonctionnel, dans les formes les plus sévères, conduit à penser que des séquelles pourraient persister plusieurs mois après la sortie d'hospitalisation. Les premiers retours d'expérience de Chine faisaient état, dès février 2020, de lésions de fibrose pulmonaire $(1,2)$ qui pourraient conduire à une insuffisance respiratoire chronique.

En juin 2020, cette hypothèse nous a conduit à mettre en place au sein de notre structure un projet de suivi pneumologique pour les patients ayant été atteints de formes sévères de Covid19.

\section{Une structure particulièrement impactée}

En raison de son orientation respiratoire, le département médico-universitaire (DMU) Approches assistance respiratoire, addictologie, tabacologie, pneumologie, physiologie, réhabilitation, réanimation, oncologie, chirurgie thoracique, handicap, endoscopie, sommeil, situé au sein du groupe hospitalier universitaire Sorbonne Université de l'Assistance PubliqueHôpitaux de Paris (AP-HP), a été particulièrement impliqué dans la prise en charge des patients atteints de Covid-19 en pneumologie, en soins intensifs et en médecine intensive et réanimation, au sein des hôpitaux de la Pitié-Salpêtrière et Tenon. 
En raison de l'afflux massif de patients, la demande de suivi pneumologique a rapidement dépassé l'offre de soins et représentait une difficulté pour organiser cette activité nouvelle dans des délais courts.

\section{Projet de suivi respiratoire impliquant un infirmier en pratique avancée}

En raison de ces nouveaux problèmes de santé et de la difficulté d'accueillir autant de nouveaux patients dans les cohortes de suivi de pneumologie, la cadre paramédicale du DMU a dédié à cette activité un infirmier en pratique avancée (IPA) diplômé de la structure. En effet, l'IPA est formé dans le domaine d'intervention «pathologies chroniques stabilisées, prévention et polypathologies courantes en soins primaires » qui inclut notamment l'insuffisance respiratoire chronique. Ses missions s'articulent autour d'activités d'orientation, d'éducation, de prévention et de dépistage (3) répondant à l'objectif de ce suivi.

L'IPA a ainsi été impliqué dans les réunions de préparation et de mise en place de ce projet avec les équipes soignantes et encadrantes médicales et paramédicales.

Il a été convenu que le suivi respiratoire des patients atteints par la Covid-19 se déroulerait en hospitalisation de jour (HDJ) afin de regrouper la réalisation des examens, les différentes évaluations, leur interprétation et la consultation de suivi.

Le contenu clinique de ce suivi ne pouvait être qu'empirique. Néanmoins, la Société de pneumologie de langue française a publié en mai 2020 un guide précisant le contenu minimal du suivi, adapté au terrain des patients (4). La prise en charge en HDJ a été adaptée à partir de ces recommandations et peut varier d'un hôpital à l'autre (Tab. 1).

\section{Le rôle de l'IPA et l'articulation avec le médecin pneumologue}

Dans le cadre de l'HDJ, l'IPA réalise certains actes du métier socle, parfois en collaboration avec une infirmière : prélèvement du bilan sanguin et éventuellement des gaz du sang artériels, enregistrement de l'électrocardiogramme, spirométrie et évaluation de la force des muscles respiratoires par mesures fonctionnelles. L'IPA procède au suivi à J90 du protocole de recherche Corimuno (étude visant à déterminer quels traitements, notamment immunomodulateurs, présentent le bénéfice-risque le plus favorable chez les adultes hospitalisés pour une infection à Sars-Cov-2 modérée à sévère). Il procède également à l'information des patients, à la recherche de leur non-opposition et à leur inclusion dans le protocole de recherche $\mathrm{RE}^{2} \mathrm{COVERI}$ (étude évaluant la récupération respiratoire au décours 
d'une infection sévère à très sévère à Sars-CoV-2). Cette dernière étude étant non interventionnelle, l'IPA est habilité à réaliser les inclusions (obtention de l'attestation de bonnes pratiques cliniques-investigateur dispensée par le groupement interrégional de recherche clinique et d'innovation d'Ile-de-France) (5).

Avant de recevoir le patient en consultation, l'IPA prend connaissance de l'histoire de la maladie au travers des éléments disponibles dans son dossier informatisé. Il recueille les résultats de l'ensemble des examens, les interprète et en évalue l'évolution.

L'IPA et le patient se rencontrent ensuite en consultation pour un entretien singulier. Cet échange est un temps privilégié pour créer une relation de confiance avec le patient. L'infirmier procède au recueil de données à travers le discours du patient et par l'interrogatoire (encadré $1)$.

Selon la situation du patient, l'IPA répond à ses interrogations et apporte des conseils éducatifs personnalisés. Il recherche l'adhésion du patient dans un processus de codécision, le plus souvent sur l'activité physique, l'alimentation et la gestion des séquelles cutanées.

L'IPA porte à la connaissance du patient les résultats de ses examens et les lui explique.

Il réalise un examen clinique et paraclinique adapté à la situation du patient (encadré 2).

Le médecin pneumologue effectue une interprétation du scanner et échange avec l'IPA en amont ou en fin de consultation. Le médecin supervise les consultations et est sollicité systématiquement lorsque la situation du patient est complexe, nécessite l'approfondissement de l'examen clinique, implique l'élaboration d'un diagnostic ou la mise en place d'un traitement. Une présentation du dossier est faite par l'IPA en amont ou en aval de la consultation, selon la situation du patient et à la demande de l'IPA ou du médecin. Selon la situation, la consultation peut même être coconduite par l'IPA et le médecin.

L'IPA établit des conclusions cliniques ; il les réalise soit en autonomie, soit en collaboration avec le médecin lorsque la situation est complexe. Il prescrit des examens complémentaires, programme le prochain suivi et oriente le patient vers d'autres professionnels de santé si besoin est.

L'IPA procède enfin à la rédaction du compte-rendu d'hospitalisation $(\mathrm{CRH})$, incluant le recueil de toutes les données, les conclusions cliniques et les actions engagées. Le CRH est adressé au patient et aux médecins impliqués dans le parcours du patient. Il est contresigné par le médecin 
en charge du patient, puis archivé dans le dossier médical informatisé de l'AP-HP, ce qui le rend accessible à tout professionnel disposant des accès nécessaires qui aurait besoin d'en prendre connaissance.

Ce processus garantit la protection des données du patient et la circulation de l'information aux professionnels de santé impliqués dans le parcours du patient.

\section{Un retour d'expérience}

Cette activité de suivi post-Covid-19 a été riche pour l'IPA.

En effet, elle représente sa première activité clinique en pratique avancée. Elle a été l'occasion de débuter la mobilisation des compétences acquises lors de la formation et d'approfondir ses connaissances, mais aussi de développer de la collaboration et de l'échange entre médecins et IPA.

Cette activité a présenté un intérêt particulièrement fort dans le cadre de la Covid-19 puisqu'il s'agit d'une maladie nouvelle, dont l'état des connaissances concernant ses potentielles séquelles était plutôt empirique et déductif.

Elle permet de répondre à des besoins de santé nouveaux et à un afflux de patients qui a été particulièrement dense.

La majorité des pneumologues expriment une satisfaction concernant ce mode de collaboration dans lequel leur expertise est sollicitée, mais aussi leurs qualités pédagogiques. Les patients ont exprimé leur satisfaction vis-à-vis de ce type de consultation, où ils ont pu bénéficier d'un temps d'échange lors duquel ils étaient écoutés. Ils pouvaient obtenir des réponses honnêtes et étaient particulièrement satisfaits que les résultats de leurs examens soient expliqués d'une façon intelligible.

L'IPA impliqué dans ce projet a exprimé sa satisfaction, ayant le sentiment de gagner beaucoup en matière de relations interpersonnelles, tant avec les patients qu'avec les médecins ou les équipes administratives et soignantes des HDJ.

Tableau 1. Suivi clinique en hospitalisation de jour post-Covid-19 dans les hôpitaux parisiens Tenon et la PitiéSalpêtrière

\begin{tabular}{|c|c|}
\hline Hôpital Tenon & Hôpital de la Pitié-Salpêtrière \\
\hline$-\quad$ Scanner thoracique non injecté & - Scanner thoracique non injecté \\
\hline
\end{tabular}




\begin{tabular}{|c|c|}
\hline $\begin{array}{ll}\text { - } & \text { Bilan biologique } \\
\text { - } & \text { Electrocardiogramme } \\
\text { - } & \text { Explorations fonctionnelles } \\
\text { respiratoires incluant spirométrie, } \\
\text { pléthysmographie, mesure de la } \\
\text { diffusion, test de marche de } 6 \\
\text { minutes et évaluation de la force des } \\
\text { muscles respiratoires } \\
\text { Consultation IPA avec validation } \\
\text { médicale }\end{array}$ & $\begin{array}{ll}\text { - } & \text { Bilan biologique } \\
\text { - } & \text { Explorations fonctionnelles } \\
\text { respiratoires incluant spirométrie, } \\
\text { mesure de la diffusion, test de } \\
\text { marche de } 6 \text { minutes et évaluation de } \\
\text { la force des muscles respiratoires } \\
\text { - } \quad \text { Evaluation psychologique } \\
\text { - Consultation IPA avec validation } \\
\text { médicale }\end{array}$ \\
\hline
\end{tabular}

IPA : infirmier en pratique avancée

Encadré 1. Contenu de l'interrogatoire

- Description du mode de vie: famille, soutien social, lieu de vie, profession, tabagisme, consommation d'alcool ou autres addictions, exposition environnementale, autonomie avant la maladie.

- Complément à l'histoire de la maladie (recherche des informations non disponibles dans le dossier informatisé : consultations médicales ou hospitalisation hors AP-HP, séjour en soins de suite et de réadaptation, etc.).

- Recherche des symptômes séquellaires de l'atteinte Covid-19 ou de l'hospitalisation : asthénie, dyspnée, toux, douleurs thoraciques, myalgies, anosmie et dysgueusie, perte de poids et son évolution, éruption cutanée, vertiges, malaises, anxiété et dépression, sommeil et troubles sexuels.

- Description et gradation de la dyspnée en utilisant le score Modified medical research council.

- Retentissement des symptômes : capacité à réaliser les actes de la vie quotidienne, autonomie, reprise du travail, activité physique, rééducation, anorexie, consommation de soins, troubles locomoteurs.

Encadré 2. Examen clinique et paraclinique

- Mesure des paramètres vitaux : pression artérielle, fréquence cardiaque, saturation pulsée en oxygène, poids, taille, calcul de l'indice de masse corporelle.

- Recherche d'amyotrophie.

- Auscultation pulmonaire et cardiaque. 
- Test de la force musculaire par le score Medical research council.

- Recherche de signes de phlébite et d'insuffisance cardiaque droite.

- Evaluation des séquelles cutanées (escarres, lésions de cordon d'intubation, de décubitus ventral, etc.).

- Orifice de trachéotomie, recherche de stridor.

- Recherche de signes de syndrome d'apnées du sommeil par le score d'Epworth. 


\section{Références :}

1. Ye Z, Zhang Y, Wang Y, Huang Z, Song B. Chest CT manifestations of new coronavirus disease 2019 (COVID-19): a pictorial review. Eur Radiol. août 2020;30(8):4381-9.

2. Pan Y, Guan H, Zhou S, Wang Y, Li Q, Zhu T, et al. Initial CT findings and temporal changes in patients with the novel coronavirus pneumonia (2019-nCoV): a study of 63 patients in Wuhan, China. Eur Radiol. juin 2020;30(6):3306-9.

3. LOI n ${ }^{\circ} 2016-41$ du 26 janvier 2016 de modernisation de notre système de santé - Article 119. 2016-41 janv 26, 2016.

4. Andrejak C, Blanc F-X, Costes F, Crestani B, Debieuvre D, Perez T, et al. Guide pour le suivi respiratoire des patients ayant présenté une pneumonie à SARS-CoV-2. Propositions de prise en charge élaborées par la Société de pneumologie de langue française. Version du 10 mai 2020. Rev Mal Respir. juin 2020;37(6):505-10.

5. Article L1122-1. Code de la santé publique. Disponible sur: https://www.legifrance.gouv.fr/codes/article_lc/LEGIARTI000037825788/ 